\title{
Benefits of different intensity of aerobic exercise in modulating body composition among obese young adults: a pilot randomized controlled trial
}

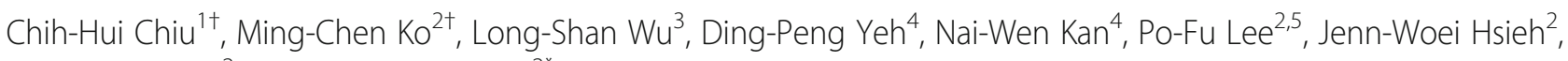
Ching-Yu Tseng ${ }^{2}$ and Chien-Chang $\mathrm{Ho}^{2^{*}}$

\begin{abstract}
Background: The aim of present study was to compare the effects of different aerobic exercise intensities and energy expenditures on the body composition of sedentary obese college students in Taiwan.

Methods: Forty-eight obese participants [body mass index (BMI) $\geq 27 \mathrm{~kg} / \mathrm{m}^{2}$, age $18-26$ years] were randomized into four equal groups $(n=12)$ : light-intensity training group (LITG), $40 \%-50 \%$ heart rate reserve (HRR); middleintensity training group (MITG), 50\%-70\% HRR; high-intensity training group (HITG), 70\%-80\% HRR; and control group (CG). The aerobic exercise training program was conducted for 60 min per day on a treadmill 3 days per week for 12 weeks. All participant anthropometric data, blood biochemical parameters, and health-related physical fitness components were measured at baseline and after 12 weeks.
\end{abstract}

Results: At baseline, the anthropometric indices did not differ significantly among the four groups $(p>0.05)$. After $12-$ week exercise intervention, the HITG and MITG had significantly more changes in body weight, waist circumference (WC), waist-to-hip ratio (WHR), and waist-to-height ratio (WHtR) than the LITG. The changes in BMI and body fat percentage differed among all four groups $(p<0.05)$.

Conclusions: A 12-week high-intensity exercise intervention with high energy expenditure can considerably reduce body weight, body fat, WC, WHR, and WHtR, whereas a light-intensity exercise intervention can significantly reduce body weight and body fat.

Trial registration: Current Controlled Trials TPECTR09831410900, registered on 24 ${ }^{\text {th }}$ Dec 2009.

Keywords: Obesity, Aerobic exercise, Exercise intensity, Body composition

\section{Background}

The percentage of the population that is obese increased in both the developed and developing countries [1]. Obesity is defined as a condition of abnormal or excessive body fat (i.e., the accumulation of adipose tissue) to the extent that health may be impaired [2]. Studies have demonstrated that obesity is linked to higher risk of developing various chronic diseases, such as cardiovascular

\footnotetext{
* Correspondence: 093703@mail.fju.edu.tw

${ }^{\dagger}$ Equal contributors

${ }^{2}$ Department of Physical Education, Fu Jen Catholic University, New Taipei

City 24205, Taiwan

Full list of author information is available at the end of the article
}

disease, type 2 diabetes mellitus, and cancers [3, 4]. According to the clinical standards for defining obesity proposed by Taiwan's Ministry of Health and Welfare (MOHW), children and adolescents are defined as obese based on their body mass index (BMI) over age- and sex-specific 95th percentile values; by contrast, adults with a BMI $\geq 27 \mathrm{~kg} / \mathrm{m}^{2}$ are defined as being obese.Nevertheless, the standards for "obesity" in adults are also diverse among different countries. For example, the World Health Organization (WHO)-Asian standard for obesity in adults is a BMI $\geq 25 \mathrm{~kg} / \mathrm{m}^{2}$, in contrast to the obesity standard set by the MOHW in Taiwan [5]. The MOHW's standard is the more frequently used 
anthropometric measure to evaluate the degree of obesity among Taiwanese adults. Based on data from the three waves of the Nutrition and Health Surveys in Taiwan (NAHSIT), which occurred in 1993-1966, 20052008, and 2013-2014, Chang et al. [6] noted that the prevalence of adult obesity has drastically increased in recent decades from $11.8 \%$ to $17.9 \%$ to $22.1 \%$, respectively. Two earlier surveys were conducted using multiple stage sampling among Taiwanese college students, wherein the prevalence of overweight and obesity was $31.4 \%$ in men and $16.4 \%$ in women $[7,8]$. Weight loss is crucial for improving health and reducing body fat [9]. Thus, considering the public health, weight loss is effective for reducing chronic disease risk [10].

Aerobic exercise increases peak oxygen consumption ( $\mathrm{VO}_{2}$ peak), which is closely correlated with total body fat percentage (BF\%); aerobic exercise is also a powerful strategy for weight loss, particularly body fat loss [11, 12]. When designing a suitable weight loss program, exercise duration and intensity are generally manipulated. Moderate aerobic exercise for at least $150 \mathrm{~min}$ per week may improve risk factors for metabolic syndrome like body composition, insulin resistance and glycated haemoglobin (HbA1c) [13]. American College of Sports Medicine suggested that long-term moderate aerobic exercise for $>150$ or $200-300$ min per week can significantly reduce body weight when the diet is not controlled [10]. However, when exercise intensities differ, exercise expenditure is not the only factor responsible for weight loss [14]. The effects of increasing exercise intensity on weight loss when exercise duration is kept constant remain unknown.

High-intensity exercise training may effectively reduce body and abdominal fat [15]. When energy expenditure is held equal, high-intensity exercise is more beneficial for improving body composition and reducing abdominal fat than low-intensity exercise $[15,16]$. Furthermore, a randomized controlled trial reported that highintensity interval training (which resulted in low energy expenditure) was found equally effective in reducing body weight and body fat as was low-intensity endurance exercise training [17]. This finding suggests that a higher exercise intensity may be more effective in improving body composition. However, long-term evidence from randomized controlled trials on the effect of exercise duration with different intensities and energy expenditures on body composition is scant. Furthermore, using a single duration during aerobic exercise training can increase the appetite of obesity people during the recovery period [18]. Moreover, the effects of long-term aerobic exercise training on the appetite and weight loss remain unknown. Therefore, in this pilot randomized controlled trial, we compared the effect of different aerobic exercise intensities and unequal energy expenditures on body composition among sedentary obese college students in Taiwan.

\section{Material and methods Study design and participants}

We conducted a 12-week randomized controlled trial. A total of 58 obese sedentary college students (aged 1826 years) were recruited using advertisements that were posted in the Fitness Center of Chung Hua University in Taiwan. Of these 58 voluntary participants, five did not meet the inclusion criteria; the remaining 53 were randomly assigned into a light-intensity training group (LITG, $n=13$ ), middle-intensity training group (MITG, $n=13$ ), high-intensity training group (HITG, $n=13$ ), and control group (CG, $n=14$ ) following initial screening. The inclusion criteria for the recruitment and enrollment of participants were as follows: 1) BMI $\geq 27 \mathrm{~kg} / \mathrm{m}^{2}$, 2) exercise comprising $\leq 1$ session per week with $\leq 30 \mathrm{~min}$ at moderate-to-vigorous intensity, and 3) a fitness performance level that allowed the completion of the aerobic exercise training in present study. However, five participants dropped out of the study because of ill health, lack of availability, and family commitments. Finally, 48 participants (34 men and 14 women)-with 12 in each group-were evaluated further (Fig. 1). To reduce the influence of other confounding factors, participants with a cardiovascular disease, diabetes, liver dysfunction, renal impairment, a endocrine disorder, a smoking habit, and weight-loss pill consumption were excluded. The experimental protocol was approved by the institutional review board of Taipei Physical Education College, and informed consent was obtained from each participant after fully explaining the study.

\section{Exercise intervention}

The exercise programs, comprising three 60 -min sessions weekly for 12 weeks, progressing gradually in intensity were conducted at the Fitness Center of Chung Hua University (Fig. 1). Each session included 10-min warm-up, cool-down, and stretching periods. The LITG performed the aerobic exercise for $40 \%-50 \%$ heart rate reserve (HRR) during weeks $1-12$, as determined by using a walking treadmill exercise test. The MITG performed the aerobic exercise for $40 \%-50 \%$ HRR during weeks $1-6$, increasing to $50 \%-70 \%$ HRR during weeks $7-12$. The HITG performed the aerobic exercise for $40 \%-50 \%$ HRR during weeks $1-6$, increasing to $70 \%-$ $80 \%$ HRR during weeks 7-12. Heart rate was monitored continuously on a Polar Accurex monitor (Kempele, Finland) for adjusting workload to achieve the target heart rate. During the 12-week period, all participants were asked not to change their dietary habits; moreover, the CG was asked to not change their exercise habits and maintain normal activity. 


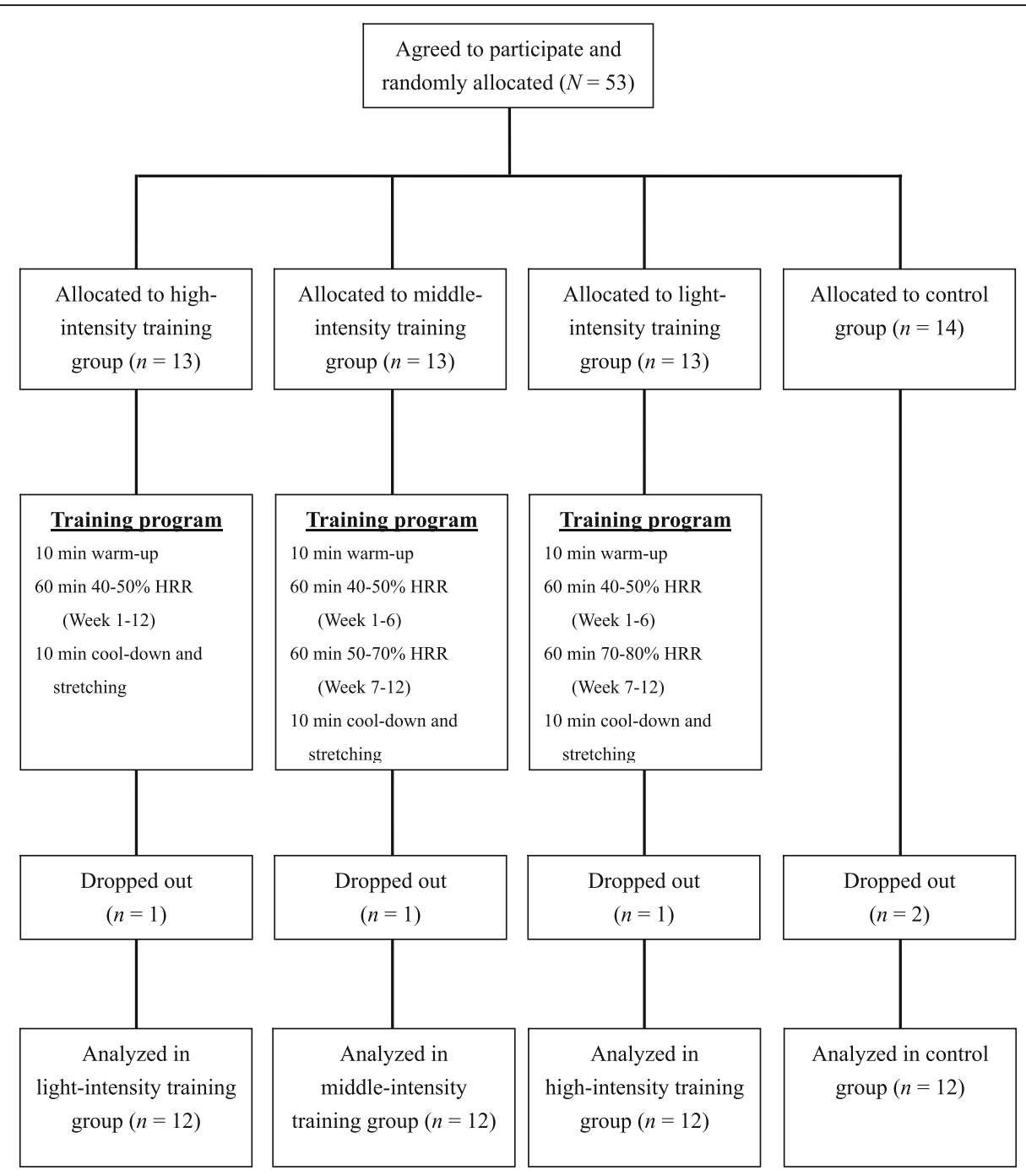

Fig. 1 Flow chart of participant enrollment and completion of the 12-week aerobic exercise program

\section{Experimental procedure}

Age, sex, smoking habit, current alcohol consumption, and family history of chronic diseases of all participants were recorded. Systolic and diastolic blood pressure after a resting period of at least $5 \mathrm{~min}$ was obtained from the right arm through auscultation by using a mercury sphygmomanometer (Baumanometer, Copiague, NY, USA). All participants were instructed to maintain their typical diet and daily physical activity throughout the study period, and compliance with this instruction was assessed through a 24-h diet recall questionnaire and 7-day International Physical Activity Questionnaire administered at the beginning and end of the study. The validity and reliability of these questionnaires have been described elsewhere [19, 20]. Other than diet and physical activity, total energy intake or estimated energy expenditure did not change significantly over the study period (data not shown). Participant demographic data, anthropometric measurements, blood biochemical parameters, and health-related physical fitness components were assessed at baseline and Week 12.

\section{Health-related physical fitness assessment}

The National Physical Fitness Survey conducted by the National Council on Physical Fitness and Sports in Taiwan during 1999-2001 and 2011-2013 included four main components of health-related physical fitness: cardiorespiratory endurance, muscle strength and endurance, flexibility, and body composition. These tests have been used to estimate the health-related physical fitness of people aged 20-65 years and can be performed by research assistants who have attended the official training seminar and passed the certification test on standardized procedures. In present study, body composition and its related outcomes such as cardiorespiratory endurance were measured, which are detailed as follows [21-23]: 
- Body composition: Body composition was determined using six anthropometric indices including BMI, WC, hip circumference (HC), waistto-hip ratio (WHR), waist-to-height ratio (WHtR), and $\mathrm{BF} \%$. These measurements were performed after the participants removed their shoes and heavy clothing. The values of body height and weight were recorded in meters to the nearest $0.1 \mathrm{~cm}$ and $0.1 \mathrm{~kg}$ with a calibrated automatic height and weight scale (model HW686, Taiwan), respectively. BMI was calculated using the following formula: BMI = body weight (in $\mathrm{kg}$ )/[height (in $\mathrm{m}$ ) $]^{2}$. WC was measured to the nearest $0.1 \mathrm{~cm}$ by using a flexible steel tape at the level midway between the lowest rib margin and the iliac crest, and $\mathrm{HC}$ was measured at the widest level over the greater trochanters. WHR was calculated as WC divided by $\mathrm{HC}$, and WHtR was calculated as WC divided by height. BF\%, fat mass, and fat-free mass was determined through bioelectrical impedance analysis by using a Body Composition Analyzer (TANITA Corporation, Japan).

- Cardiorespiratory endurance: Cardiorespiratory endurance was measured using a standardized 3-min step test (step height $35 \mathrm{~cm}$; frequency 24 steps $/ \mathrm{min}$ ). When the exercise was completed, the participants were immediately seated and their heart rates were measured for $1 \mathrm{~min}$, starting within $5 \mathrm{~s}$ of the end of the exercise. The sum of the heart rates during the recovery period was compared with the sum of the heart rates during three periods after the test $-1-1.5$, $2-2.5$, and 3-3.5 min [24, 25]. The test was terminated if a subject lost balance, missed the stepping rhythm for three steps, or reported any discomfort during the test [26]. The cardiorespiratory endurance index $(\mathrm{CEI})$ was calculated as $\mathrm{CEI}=$ [duration of exercise $(\mathrm{s}) \times 100] /[$ sum of heart rates during the recovery period/2]. Although maximal exercise testing is a sophisticated measure of cardiorespiratory endurance, generally not feasible or desirable clinically. By contrast, step tests are submaximal exercise tests requiring minimal equipment. Various step tests (different step heights and stepping rates) estimate $\mathrm{VO}_{2}$ peak and are reliable and valid measures of cardiopulmonary fitness $[27,28]$. The 3-min step test used in our study was adapted from the Harvard Step Test, which assesses cardiorespiratory endurance based on the speed of heart-rate recovery from submaximal exercise. The CEI of the 3-min step test was positively correlated $(r=0.50)$ with $\mathrm{VO}_{2}$ peak in healthy Taiwanese adults [29].

The content of the measurements was explained to each participant, and then, they were given a $10-\mathrm{min}$ warm-up and stretching period to achieve their most favorable performance. These measurements were scheduled in the morning before any other exercise. All measurements were performed by the same well-trained investigator. Each measurement was obtained two times, and the average of the two values for each test was used.

\section{Blood sample analysis}

Following a 12-h overnight fasting period, venous blood samples $(15 \mathrm{~mL})$ were obtained from an antecubital vein in the sitting position after a 20-min rest between 07:00 a.m. and 09:00 a.m. at baseline and Week 12. The blood was immediately transferred into vacutainer tubes (Becton Dickinson, Rutherford, NJ, USA) containing or not containing $0.1 \%$ EDTA as the anticoagulant for estimating the hematological status. Serum or plasma was separated through centrifugation at $2500 \mathrm{rpm}$ for 15 min at $4{ }^{\circ} \mathrm{C}$ and then stored at $-80{ }^{\circ} \mathrm{C}$ until analysis. Hematological entities including fasting glucose, total cholesterol (TC), low-density lipoprotein cholesterol (LDL-C), high-density lipoprotein cholesterol (HDL-C), and triglycerides (TG) were measured using an automated biochemical analyzer. The analytical inter- and intra-assay coefficients of variation obtained in our laboratory were respectively as follows: fasting glucose, $2.4 \%$ and $1.6 \%$; TC, $1.3 \%$ and $0.7 \%$; LDL-C, $2.2 \%$ and 1.6\%; HDL-C, $4.5 \%$ and $1.6 \%$; and TG, $2.1 \%$ and $1.3 \%$.

\section{Statistical analysis}

All analyses were performed using SAS software package (version 9.12, Statistical Analysis System, SAS Institute Inc., Cary, NC, USA). Differences in the participant demographic data, anthropometric measurement, blood biochemical parameters, and health-related physical fitness components between baseline and Week 12 were analyzed using a paired $t$ test. Comparisons between the mean values of normally distributed variables between groups of exercise were analyzed using one-way analysis of variance (ANOVA). When a significant $F$ value $(p<0.05)$ was noted, Tukey's post hoc test was performed to determine the differences between the pairs of means. The relationship between changes in various anthropometric indices and physical fitness measurements were examined using Pearson partial correlation coefficients after adjustment for potential confounders. All data are expressed as means \pm standard errors (SEs) or frequency percentages (\%).We considered a significance level of $p<0.05$ to reject the null hypothesis. Since this is a pilot study, we did not calculate sample size.

\section{Results}

Baseline characteristics of the four groups including the demographic data, anthropometric measurement, blood biochemical parameters, and health-related physical fitness components did not differ significantly (all $p>0.05$; Table 1). 
Table 1 Baseline characteristics of subjects

\begin{tabular}{|c|c|c|c|c|c|}
\hline Characteristic & $\operatorname{HITG}(n=12)$ & MITG $(n=12)$ & LITG $(n=12)$ & CG $(n=12)$ & $P$-value \\
\hline Age (years) & $21.75 \pm 0.74$ & $20.92 \pm 0.38$ & $20.67 \pm 0.56$ & $20.83 \pm 0.71$ & 0.603 \\
\hline Gender (Male \%) & 8/4 (66.67) & 9/3 (75.00) & 8/4 (66.67) & 9/3 (75.00) & 0.318 \\
\hline Height (cm) & $169.17 \pm 2.46$ & $170.09 \pm 2.84$ & $168.20 \pm 2.57$ & $171.40 \pm 2.42$ & 0.840 \\
\hline Body weight (kg) & $84.38 \pm 2.71$ & $92.12 \pm 3.89$ & $87.53 \pm 4.62$ & $89.83 \pm 4.38$ & 0.562 \\
\hline BMI $\left(\mathrm{kg} / \mathrm{m}^{2}\right)$ & $29.43 \pm 0.56$ & $31.78 \pm 0.99$ & $30.70 \pm 0.99$ & $30.38 \pm 0.86$ & 0.305 \\
\hline$W C(\mathrm{~cm})$ & $97.42 \pm 2.08$ & $103.83 \pm 2.65$ & $99.38 \pm 2.50$ & $98.79 \pm 2.86$ & 0.322 \\
\hline $\mathrm{HC}(\mathrm{cm})$ & $109.96 \pm 1.66$ & $115.25 \pm 1.97$ & $113.58 \pm 2.44$ & $111.83 \pm 1.69$ & 0.273 \\
\hline WHR & $0.89 \pm 0.01$ & $0.90 \pm 0.01$ & $0.88 \pm 0.02$ & $0.88 \pm 0.01$ & 0.680 \\
\hline $\mathrm{WHtR}$ & $0.58 \pm 0.01$ & $0.61 \pm 0.02$ & $0.59 \pm 0.01$ & $0.58 \pm 0.01$ & 0.178 \\
\hline Body fat (\%) & $34.50 \pm 1.78$ & $38.55 \pm 2.09$ & $39.89 \pm 2.40$ & $33.32 \pm 1.07$ & 0.054 \\
\hline Fat mass (kg) & $29.04 \pm 1.70$ & $35.35 \pm 2.24$ & $35.02 \pm 2.97$ & $30.03 \pm 1.99$ & 0.115 \\
\hline Fat-free mass (kg) & $55.34 \pm 2.47$ & $56.77 \pm 3.34$ & $52.51 \pm 3.53$ & $59.80 \pm 2.77$ & 0.408 \\
\hline SBP (mmHg) & $126.17 \pm 2.23$ & $127.75 \pm 2.54$ & $119.67 \pm 4.24$ & $126.08 \pm 1.64$ & 0.203 \\
\hline $\mathrm{DBP}(\mathrm{mmHg})$ & $75.75 \pm 1.87$ & $78.42 \pm 2.87$ & $79.25 \pm 3.08$ & $83.75 \pm 2.59$ & 0.206 \\
\hline Fasting glucose (mg/dL) & $89.42 \pm 2.53$ & $88.67 \pm 2.33$ & $87.83 \pm 2.93$ & $86.92 \pm 2.77$ & 0.919 \\
\hline $\mathrm{TC}(\mathrm{mg} / \mathrm{dL})$ & $184.67 \pm 3.93$ & $185.75 \pm 3.67$ & $181.08 \pm 4.30$ & $181.50 \pm 3.87$ & 0.795 \\
\hline $\mathrm{HDL}-\mathrm{C}(\mathrm{mg} / \mathrm{dL})$ & $48.67 \pm 2.82$ & $44.42 \pm 1.60$ & $47.25 \pm 2.67$ & $47.17 \pm 1.86$ & 0.619 \\
\hline LDL-C (mg/dL) & $116.92 \pm 3.06$ & $120.83 \pm 3.75$ & $113.67 \pm 3.85$ & $111.67 \pm 4.12$ & 0.334 \\
\hline TC/HDL-C ratio & $3.50 \pm 0.16$ & $4.31 \pm 0.20$ & $3.83 \pm 0.31$ & $3.71 \pm 0.25$ & 0.112 \\
\hline TG (mg/dL) & $98.50 \pm 3.96$ & $114.17 \pm 9.94$ & $96.42 \pm 11.19$ & $105.67 \pm 9.30$ & 0.506 \\
\hline
\end{tabular}

Abbreviations: BMI body mass index, CEI cardiorespiratory endurance index, CG control group, DBP diastolic blood pressure, HC hip circumference, HITG highintensity training group, LITG light-intensity training group, MITG moderate-intensity training group, SBP systolic blood pressure, SE standard error, TG triglycerides, WC waist circumference, WHR waist-to-hip ratio, WHtR waist-to-height ratio. Data are expressed as mean \pm SE. Groups compared using one-way ANOVA with Tukey's post hoc comparisons ( $P$-values shown)

The changes in the health-related physical fitness components including those in body composition and cardiorespiratory fitness among the four groups between baseline and Week 12 are presented in Table 2. At Week 12, the HITG exhibited significant improvement in body composition in body composition and cardiorespiratory fitness measurements $(p \leq 0.05)$. Similarly, the body composition and cardiorespiratory fitness measurements significantly improved in the MITG and LITG; in addition, no differences in fat-free mass were observed in the MITG and LITG and no significant differences in HC, fat-free mass, and sit-and-reach test in the LITG. No significant changes were observed in any of these outcome measurements in the CG.

Table 3 also presents the differences in the changes in health-related physical fitness components among the four groups. ANOVA indicated significant means group effects for changes in the body weight, BMI, WC, HC, WHR, WHtR, \%BF, fat mass, and CEI (all $p<0.05$ ); however, the changes in fat-free mass did not have significant differences in the group effects. Furthermore, post hoc comparisons revealed that the changes in body weight, WC, WHR, WHtR, fat mass, and CEI were significantly higher in the HITG and MITG compared with the LITG and CG; changes in the HITG and MITG did not differ significantly, whereas those in the LITG and CG did. In addition, the changes in $\mathrm{BMI}$ and $\mathrm{BF} \%$ significantly differed among the four groups.

Moreover, pooled partial Pearson correlation analyses ( $n=48$ ) were conducted to examine the relationship among changes in cardiorespiratory fitness and body composition after adjusting for age and sex (Table 4). The changes in CEI were negatively associated with changes in body weight, BMI, WC, HC, WHR, WHtR, $\% \mathrm{BF}$, fat mass, and fat-free mass $(p<0.05)$.

\section{Discussion}

In this randomized controlled trial, we examined the effect of aerobic exercise at three intensities on body weight and body fat of obese individuals. After a 12week exercise intervention, higher intensity exercise training (HITG and MITG) led to significantly more changes in body composition. Body weight, WC, WHR, and WHtR were significantly improved in the HITG and MITG compared with those in the LITG and CG. Furthermore, low-intensity exercise intervention without diet control also improved body composition. This study emphasizes that regular exercise training is a major 


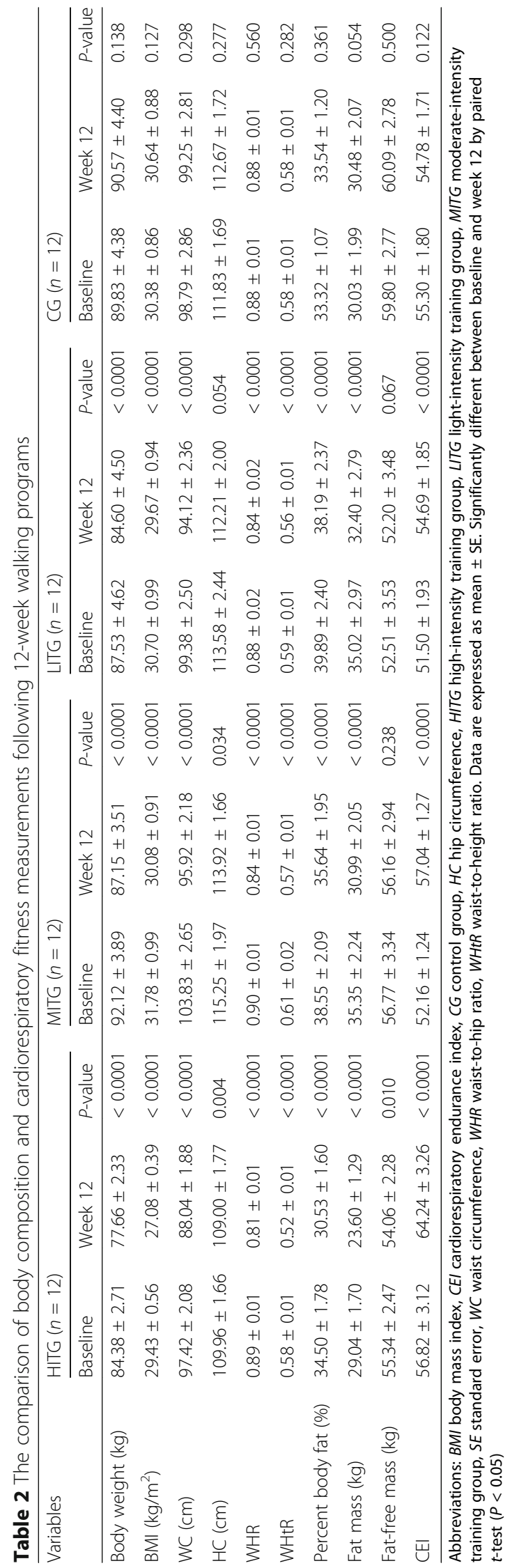


Table 3 Changes in body composition and cardiorespiratory fitness measurements following 12-week walking programs

\begin{tabular}{|c|c|c|c|c|c|c|}
\hline Variables & $\operatorname{HITG}(n=12)$ & MITG $(n=12)$ & $\operatorname{LITG}(n=12)$ & CG $(n=12)$ & $P$-value & Tukey's post hoc test \\
\hline Body weight (kg) & $-6.73 \pm 0.67$ & $-4.97 \pm 0.46$ & $-2.93 \pm 0.19$ & $0.74 \pm 0.46$ & $<0.0001$ & HITG, MITG > LITG > CG \\
\hline BMI $\left(\mathrm{kg} / \mathrm{m}^{2}\right)$ & $-2.34 \pm 0.24$ & $-1.70 \pm 0.13$ & $-1.04 \pm 0.06$ & $0.26 \pm 0.16$ & $<0.0001$ & HITG $>$ MITG $>$ LITG $>C G$ \\
\hline$W C(\mathrm{~cm})$ & $-9.38 \pm 0.74$ & $-7.92 \pm 0.69$ & $-5.25 \pm 0.23$ & $0.46 \pm 0.42$ & $<0.0001$ & HITG, MITG $>$ LITG $>$ CG \\
\hline $\mathrm{HC}(\mathrm{cm})$ & $-0.96 \pm 0.26$ & $-1.33 \pm 0.55$ & $-1.38 \pm 0.64$ & $0.83 \pm 0.73$ & 0.028 & MITG, LITG > CG \\
\hline WHR & $-0.08 \pm 0.01$ & $-0.06 \pm 0.01$ & $-0.04 \pm 0.004$ & $-0.002 \pm 0.004$ & $<0.0001$ & HITG, MITG > LITG > CG \\
\hline $\mathrm{WHtR}$ & $-0.06 \pm 0.004$ & $-0.05 \pm 0.004$ & $-0.03 \pm 0.001$ & $0.003 \pm 0.002$ & $<0.0001$ & HITG, MITG > LITG > CG \\
\hline Percent body fat (\%) & $-3.97 \pm 0.28$ & $-2.91 \pm 0.25$ & $-1.70 \pm 0.14$ & $0.23 \pm 0.24$ & $<0.0001$ & HITG $>$ MITG $>$ LITG $>$ CG \\
\hline Fat mass (kg) & $-5.44 \pm 0.49$ & $-4.35 \pm 0.23$ & $-2.62 \pm 0.22$ & $0.45 \pm 0.21$ & $<0.0001$ & HITG, MITG > LITG > CG \\
\hline Fat-free mass (kg) & $-1.29 \pm 0.42$ & $-0.61 \pm 0.49$ & $-0.31 \pm 0.15$ & $0.29 \pm 0.42$ & 0.050 & HITG $>$ CG \\
\hline CEI & $7.42 \pm 0.34$ & $4.88 \pm 0.28$ & $3.19 \pm 0.16$ & $-0.52 \pm 0.31$ & $<0.0001$ & HITG, MITG $>$ LITG $>$ CG \\
\hline
\end{tabular}

Abbreviations: BMI body mass index, CEI cardiorespiratory endurance index, CG control group, HC hip circumference, HITG high-intensity training group, LITG lightintensity training group, MITG moderate-intensity training group, SE standard error, WC waist circumference, WHR waist-to-hip ratio, WHtR waist-to-height ratio. Data are expressed as mean \pm SE. Groups compared using one-way ANOVA with Tukey's post hoc comparisons $(P<0.05)$

factor responsible for improving body composition and preventing abdominal obesity-related chronic disease in overweight and obese people.

Our results are consistent with those of previous studies: high-intensity training is more effective in decreasing $\mathrm{BF} \%$ [15]. The higher exercise intensity and energy expenditure may be involved in improving body composition [30, 31]. Compared with low-intensity exercise, high-intensity exercise may reduce body weight and body fat significantly, when the energy expenditure is equal $[15,16]$. The possible explanations for the effect of exercise intensity on body composition control are that high intensity exercise can increase catecholamine and growth hormone release [32, 33], postexercise oxygen consumption [34, 35], and lipoprotein lipase activity [36]. By contrast, higher energy expenditure during exercise can cause greater body fat loss [30, 31]. Taken

Table 4 Correlates of changes in body composition measurements with change in cardiorespiratory fitness

\begin{tabular}{lll}
\hline Variables & $\Delta$ CEl & \\
\cline { 2 - 3 } & $r$ & $P$-value \\
\hline$\Delta$ Body weight $(\mathrm{kg})$ & -0.857 & $<0.0001$ \\
$\Delta$ BMl $\left(\mathrm{kg} / \mathrm{m}^{2}\right)$ & -0.848 & $<0.0001$ \\
$\Delta$ WC $(\mathrm{cm})$ & -0845 & $<0.0001$ \\
$\Delta$ HC $(\mathrm{cm})$ & -0.345 & 0.019 \\
$\Delta$ WHR & -0.798 & $<0.0001$ \\
$\triangle$ WHtR & -0.841 & $<0.0001$ \\
$\triangle$ Percent body fat (\%) & -0.843 & $<0.0001$ \\
$\triangle$ Fat mass (kg) & -0.871 & $<0.0001$ \\
$\triangle$ Fat-free mass (kg) & -0.442 & 0.002 \\
\hline
\end{tabular}

Abbreviations: $B M I$ body mass index, CEl cardiorespiratory endurance index, CG control group, HC hip circumference, HITG high-intensity training group, LITG light-intensity training group, MITG moderate-intensity training group, WC waist circumference, $W H R$ waist-to-hip ratio, WHtR waist-to-height ratio. $\Delta$, change in. Data are expressed as Pearson correlation coefficients after adjusting for age and gender $(P<0.05)$. Data derived from pooled groups $(N=48)$ together, higher exercise intensity may result in significantly higher long-term reduction in body fat compared with lower exercise intensity.

Previous studies examining the effect of exercise training on appetite perceptions during postexercise recovery have reported inconsistent results [37-42]. The present study demonstrated that aerobic exercise training may not affect appetite perceptions when the energy intake is not controlled. This finding is consistent with recent studies showing that long-term exercise training may not affect energy intake and reduce the benefit of weight loss $[37,39]$. Studies have also suggested that highintensity exercise results in reducing energy intake after exercise [41, 42]. No association between energy expenditure during exercise and energy intake or appetite perceptions was reported previously [37, 40, 41]. In present study, no such association was observed over the study period: high-intensity exercise training with more energy expenditure effectively improved body composition. Our findings indicate that high-intensity exercise training with higher energy expenditure is more effective in improving body composition than low-intensity exercise training, even when the diet is not controlled.

The differences in exercise intensities resulted in significant changes in body weight, WC, WHR, and WHtR (HITG and MITG > LITG > CG) among our participants. Thus, intensity may be crucial for reducing abdominal fat and sequentially reducing WC, WHR, and WHtR. Higher exercise intensity, but not high energy expenditure, may significantly reduce whole body fat, abdominal fat, subcutaneous abdominal fat, and abdominal visceral fat compared with lower exercise intensity $[15,16]$. By contrast, energy expenditure during aerobic exercise training is only associated with visceral fat and not subcutaneous or abdominal fat $[31,43]$. Although not statistically significant, the values of reduction in WC, WHR, and WHtR was higher in the HITG than in the MITG. Thus, exercise 
intensity is crucial for modulating abdominal fat. Furthermore, the additional benefit of abdominal fat reduction may be counteracted if the exercise intensity is higher than $50 \%-80 \%$ HRR.

The results of present study implicate that low-intensity exercise can also significantly reduce body weight and body fat: body weight $(-2.9 \pm 0.2 \mathrm{~kg}, p<0.001)$ and body fat $(-1.7 \% \pm 0.1 \%)$ decreased significantly in the LITG. Exercise training for 150-250 min per week effectively reduces body weight. Exercise at the similar duration and intensity can also reduce body weight and body fat [44]. These findings are consistent with those of our study, suggesting that three sessions of light-intensity training for $60 \mathrm{~min}$ per day effectively reduces body weight and body fat in sedentary obese people.

The main limitation of the present study was that we only collected data before and after the exercise intervention. Hence, the speed of weight loss during the study is unknown. Additionally, the exercise intervention was only 12 weeks in duration, and therefore was not suitable for follow-up examinations. Future research must investigate the speed of weight loss (and fluctuations therein) by using a longer exercise intervention period.

\section{Conclusion}

In conclusion, higher exercise intensity and energy expenditure can significantly reduce body weight, body fat, WC, WHR, and WHtR. Light-intensity exercise training can also significantly reduce body weight and body fat. Here, a 12-week aerobic exercise program significantly modulated the anthropometric indices in obese college students, even when the diet was not controlled. Accordingly, when prescribing an aerobic exercise training program for individuals with obesity related issues, a clinical practitioner should take the intensity of the training into consideration. A high intensity aerobic exercise training program should be prescribed by a clinical practitioner for an obese individual when the individual's exercise capacity is high enough to complete the program.

\section{Abbreviations \\ ACSM: American College of Sports Medicine; ANOVA: Analysis of variance: BF\%: Body fat percentage; BMI: Body mass index; CEl: Cardiorespiratory endurance index; CG: Control group; HC: Hip circumference; HDL-C: High- density lipoprotein cholesterol; HITG: High-intensity training group; HRR: Heart rate reserve; IPAQ: International Physical Activity Questionnaire; LDL-C: Low-density lipoprotein cholesterol; LITG: Light-intensity training group; MITG: Middle-intensity training group; MOHW: Ministry of Health and Welfare; SE: Standard error; TC: Total cholesterol; TG: Triglycerides; $\mathrm{VO}_{2}$ peak: Peak oxygen consumption; WC: Waist circumference; WHO: World Health Organization; WHR: Waist-to-hip ratio; WHtR: Waist-to-height ratio}

\section{Acknowledgements}

The authors acknowledge the college students for participating in this trial. C-H. Chiu and M-C. Ko contributed equally as first author, and C-C. Ho was the corresponding author.

\section{Funding}

No external funding was sought or obtained for present study, which was undertaken for a Taipei Physical Education College Doctor in Sport Sciences dissertation.

Availability of data and materials

All relevant materials are presented in the present manuscript.

\section{Authors' contributions}

HCC participated in the design, conducted the statistical analyses, interpreted the data, and drafted the manuscript. CHC and KMC supervised the study, assisted in data interpretation, and critically reviewed the manuscript. WLS, YDP, and KNW helped in conducting the study and revising the manuscript. LPF, HJW, and TCY helped to manage and analyze the data. All authors read and approved the final manuscript.

\section{Ethics approval and consent to participate}

The study was conducted in accordance with the Declaration of Helsinki, and all procedures have been approved by the Institutional Review Board of the Taipei Physical Education College (TPECTR09831410900). Written informed consent was obtained from each subject after a full explanation of the study.

Consent for publication

Not applicable.

Competing interests

The authors declare that they have no competing interests.

\section{Publisher's Note}

Springer Nature remains neutral with regard to jurisdictional claims in published maps and institutional affiliations.

\section{Author details}

'Department of Exercise Health Science, National Taiwan University of Sport, Taichung City 40404, Taiwan. ${ }^{2}$ Department of Physical Education, Fu Jen Catholic University, New Taipei City 24205, Taiwan. ${ }^{3}$ Graduate Institute of Sports Training, University of Taipei, Taipei City 11153, Taiwan. ${ }^{4}$ Center for General Education, Taipei Medical University, Taipei City 11031, Taiwan. ${ }^{5}$ Graduate Institute of Sport Coaching Science, Chinese Culture University, Taipei City 11114, Taiwan.

Received: 30 December 2016 Accepted: 16 August 2017

Published online: 24 August 2017

\section{References}

1. Friedman JM. Obesity in the new millennium. Nature. 2000;404(6778):632-4.

2. WHO: WHO Technical Report Series 894 Obesity: Preventing and Managing The Global Epidemic. World Health Organization: Geneva, Switzerland, 2000.

3. Haslam DW, James WP. Obesity. Lancet. 2005;366(9492):1197-209.

4. Visscher TL, Seidell JC. The public health impact of obesity. Annu Rev Public Health. 2001;22:355-75.

5. Pan WH, Flegal KM, Chang HY, Yeh WT, Yeh CJ, Lee WC. Body mass index and obesity-related metabolic disorders in Taiwanese and US whites and blacks: implications for definitions of overweight and obesity for Asians. Am J Clin Nutr. 2004;79(1):31-9.

6. Chang $\mathrm{HC}$, Yang $\mathrm{HC}$, Chang HY, Yeh $\mathrm{CJ}$, Chen HH, Huang $\mathrm{KC}$, Pan WH. Morbid obesity in Taiwan: prevalence, trends, associated social demographics, and lifestyle factors. PLoS One. 2017;12(2):e0169577.

7. Wong Y, Chen SL, Chan YC, Wang MF, Yamamoto S. Weight satisfaction and dieting practices among college males in Taiwan. J Am Coll Nutr. 1999; 18(3):223-8.

8. Wong $Y$, Huang YC. Obesity concerns, weight satisfaction and characteristics of female dieters: a study on female Taiwanese college students. J Am Coll Nutr. 1999;18(2):194-200.

9. Goodpaster BH, Delany JP, Otto AD, Kuller L, Vockley J, South-Paul JE, Thomas SB, Brown J, McTigue K, Hames KC, Lang W, Jakicic JM. Effects of diet and physical activity interventions on weight loss and cardiometabolic risk factors in severely obese adults: a randomized trial. JAMA. 2010;304(16):1795-802.

10. Donnelly JE, Blair SN, Jakicic JM, Manore MM, Rankin JW, Smith BK. American College of Sports Medicine position stand. Appropriate physical 
activity intervention strategies for weight loss and prevention of weight regain for adults. Med Sci Sports Exerc. 2009;41(2):459-71.

11. Donnelly JE, Honas JJ, Smith BK, Mayo MS, Gibson CA, Sullivan DK, Lee J, Herrmann SD, Lambourne K, Washburn RA. Aerobic exercise alone results in clinically significant weight loss for men and women: midwest exercise trial 2. Obesity. 2013;21(3):E219-28.

12. Oda K, Miyatake N, Sakano N, Saito T, Miyachi M, Tabata I, Numata T. Relationship between peak oxygen uptake and regional body composition in Japanese subjects. JSHS. 2014;3(3):233-8.

13. O'Hagan C, De Vito G, Boreham CA. Exercise prescription in the treatment of type 2 diabetes mellitus: current practices, existing guidelines and future directions. Sports Med. 2013;43(1):39-49.

14. Williams CB, Zelt JG, Castellani LN, Little JP, Jung ME, Wright DC, Tschakovsky ME, Gurd BJ. Changes in mechanisms proposed to mediate fat loss following an acute bout of high-intensity interval and endurance exercise. Appl Physiol Nutr Metab. 2013;38(12):1236-44.

15. Lee MG, Park KS, Kim DU, Choi SM, Kim HJ. Effects of high-intensity exercise training on body composition, abdominal fat loss, and cardiorespiratory fitness in middle-aged Korean females. Appl Physiol Nutr Metab. 2012;37(6):1019-27.

16. Irving BA, Davis CK, Brock DW, Weltman JY, Swift D, Barrett EJ, Gaesser GA, Weltman A. Effect of exercise training intensity on abdominal visceral fat and body composition. Med Sci Sports Exerc. 2008;40(11):1863-72.

17. Tjønna AE, Lee SJ, Rognmo $\varnothing$, Stølen TO, Bye A, Haram PM, Loennechen JP, Al-Share QY, Skogvoll E, Slørdahl SA, Kemi OJ, Najjar SM, Wisløff U. Aerobic interval training versus continuous moderate exercise as a treatment for the metabolic syndrome: a pilot study. Circulation. 2008;118(4):346-54

18. George VA, Morganstein A. Effect of moderate intensity exercise on acute energy intake in normal and overweight females. Appetite. 2003;40(1):43-6.

19. Johnson-Kozlow M, Matt GE, Rock CL. Recall strategies used by respondents to complete a food frequency questionnaire: an exploratory study. J Am Diet Assoc. 2006;106(3):430-3.

20. Craig CL, Marshall AL, Sjöström M, Bauman AE, Booth ML, Ainsworth BE, Pratt M, Ekelund U, Yngve A, Sallis JF, Oja P. International physical activity questionnaire: 12-country reliability and validity. Med Sci Sports Exerc. 2003; 35(8):1381-95

21. Chen CN, Chuang LM, Wu YT. Clinical measures of physical fitness predict insulin resistance in people at risk for diabetes. Phys Ther. 2008;88(1):1355-64.

22. Taiwan National Council on Physical Fitness and Sports: National Physical Fitness Survey. National Council on Physical Fitness and Sports: Taipei, Taiwan, 1999

23. Taiwan National Council on Physical Fitness and Sports: National Physical Fitness Survey. National Council on Physical Fitness and Sports: Taipei, Taiwan, 2001

24. Bosco JS, Gustafson WF. Measurement and evaluation in physical education, fitness, and sports. Englewood Cliffs, NJ: Prentice-Hall; 1983.

25. Heyward VH. Advanced fitness assessment and exercise prescription. Champaign, IL: Human Kinetics; 1991

26. Wu YT, Chien MY, Chen SY, Lien IN. Comparisons of health-related physical fitness in different age groups. Formosan Journal of Physical Therapy. 2000; 25(6):336-43.

27. Siconolfi SF, Garber CE, Lasater TM, Carleton RA. A simple, valid step test for estimating maximal oxygen uptake in epidemiologic studies. Am J Epidemiol. 1985;121(3):382-90.

28. Watkins J. Step tests of cardiorespiratory fitness suitable for mass testing. $\mathrm{Br}$ J Sports Med. 1984;18(2):84-9.

29. Tsai MW, Chen YL, Tsai YC. Correlations between fitness indexes of 3-minute stepping test and cardiopulmonary functions during exercise in young adults. Health Promotion Science. 2006;1:5-14.

30. Ohkawara K, Tanaka S, Miyachi M, Ishikawa-Takata K, Tabata I. A doseresponse relation between aerobic exercise and visceral fat reduction: systematic review of clinical trials. Int J Obes. 2007;31(12):1786-97.

31. Ross R, Janssen I. Physical activity, total and regional obesity: dose-response considerations. Med Sci Sports Exerc. 2001;33(Suppl 6):S521-7.

32. Boutcher SH. High-intensity intermittent exercise and fat loss. J Obes. 2011; 2011:868305.

33. Kindermann W, Schnabel A, Schmitt WM, Biro G, Cassens J, Weber F. Catecholamines, growth hormone, cortisol, insulin, and sex hormones in anaerobic and aerobic exercise. Eur J Appl Physiol Occup Physiol. 1982;49(3):389-99.

34. Mann TN, Webster C, Lamberts RP, Lambert MI. Effect of exercise intensity on post-exercise oxygen consumption and heart rate recovery. Eur J Appl Physiol. 2014;114(9):1809-20.
35. Sedlock DA. Effect of exercise intensity on postexercise energy expenditure in women. Br J Sports Med. 1991;25(1):38-40.

36. Ferguson MA, Alderson NL, Trost SG, Essig DA, Burke JR, Durstine JL. Effects of four different single exercise sessions on lipids, lipoproteins, and lipoprotein lipase. J Appl Physiol. 1998;85(3):1169-74.

37. Guelfi KJ, Halse RE. Moderate-intensity exercise affects perceived hunger and fullness but not appetite-related hormones in late pregnancy. Appl Physiol Nutr Metab. 2013;38(11):1162-5.

38. Hopkins M, King NA, Blundell JE. Acute and long-term effects of exercise on appetite control: is there any benefit for weight control? Curr Opin Clin Nutr Metab Care. 2010;13(6):635-40.

39. Martins C, Stensvold D, Finlayson G, Holst J, Wisloff U, Kulseng B, Morgan L, King NA. Effect of moderate- and high-intensity acute exercise on appetite in obese individuals. Med Sci Sports Exerc. 2015;47(1):40-8.

40. Thivel D, Aucouturier J, Doucet É, Saunders TJ, Chaput JP. Daily energy balance in children and adolescents. Does energy expenditure predict subsequent energy intake? Appetite. 2013;60(1):58-64.

41. Thivel D, Metz L, Aucouturier J, Brakoniecki K, Duche P, Morio B. The effects of imposed sedentary behavior and exercise on energy intake in adolescents with obesity. J Dev Behav Pediatr. 2013;34(8):616-22.

42. Thivel D, Metz L, Julien A, Morio B, Duché P. Obese but not lean adolescents spontaneously decrease energy intake after intensive exercise. Physiol Behav. 2014;123:41-6.

43. Koo BK, Han KA, Ahn HJ, Jung JY, Kim HC, Min KW. The effects of total energy expenditure from all levels of physical activity vs. physical activity energy expenditure from moderate-to-vigorous activity on visceral fat and insulin sensitivity in obese type 2 diabetic women. Diabet Med. 2010;27(9): 1088-92.

44. Marandi SM, Abadi NG, Esfarjani F, Mojtahedi H, Ghasemi G. Effects of intensity of aerobics on body composition and blood lipid profile in obese/ overweight females. Int J Prev Med. 2013;4(Suppl 1):S118-25.

\section{Submit your next manuscript to BioMed Central and we will help you at every step:}

- We accept pre-submission inquiries

- Our selector tool helps you to find the most relevant journal

- We provide round the clock customer support

- Convenient online submission

- Thorough peer review

- Inclusion in PubMed and all major indexing services

- Maximum visibility for your research

Submit your manuscript at www.biomedcentral.com/submit
) Biomed Central 